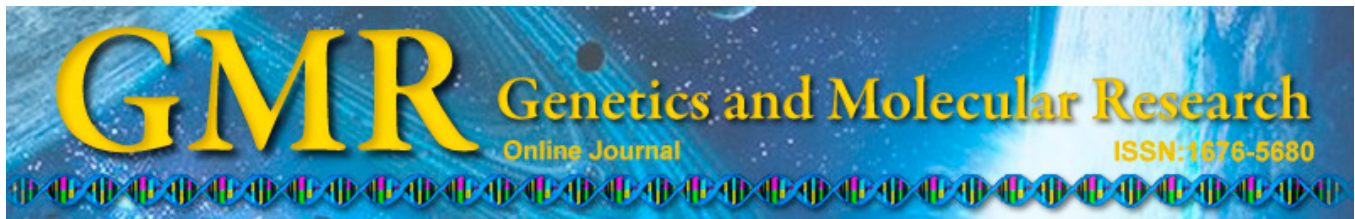

\title{
Immune protective mechanism of rMOMP protein ophthalmic vaccine regarding intraocular hypertension and retinal optic nerve injury in rats
}

\author{
X.Y. Xin ${ }^{1}$, F.Y. Sun ${ }^{2}$ and L.L. Gao ${ }^{1}$ \\ 'Department of Ophthalmology, \\ The Third Affiliated Hospital of Inner Mongolia Medical University, \\ Inner Mongolia, China \\ ${ }^{2}$ Department of Ophthalmology, Tianjin First Centre Hospital, Tianjin, China \\ Corresponding author: X.Y. Xin \\ E-mail: xinxiangyangdo@163.com
}

Genet. Mol. Res. 14 (2): 3264-3273 (2015)

Received March 25, 2014

Accepted July 28, 2014

Published April 13, 2015

DOI http://dx.doi.org/10.4238/2015.April.13.5

ABSTRACT. The aim of this study was to explore the immune
protective mechanism of rMOMP protein vaccine in intraocular
hypertension and retinal optic nerve injury in rats. The rMOMP protein
ophthalmic vaccine was prepared and quality-controlled. Sixty normal
adult SD rats were randomly divided into two groups to establish a
chronic ocular hypertension model and an optic nerve injury model.
The model rats were vaccinated with rMOMP-CS ophthalmic vaccine.
Fluorogold retrograde tracing was used to observe retinal ganglion
cells, and an immunofluorescence method to determine the expression
of retinal GAP43, CD3, BDNF, and GDNF. rMOMP protein ophthalmic
vaccine met the requirements for medicinal use. The number of retinal
ganglion cells (RGCs) of the rMOMP-CS group in the chronic ocular
hypertension model was significantly higher than that of the CS group
$(\mathrm{P}<0.05)$. The count of RGCs of the rMOMP-CS group in the optic 
nerve clamping injury model was significantly higher than that of the $\mathrm{CS}$ group $(\mathrm{P}<0.01)$. Thus, rMOMP protein ophthalmic vaccine can induce an increase in the expression of retinal neurotrophic factors, thereby exerting a protective effect on damaged retinal optic nerve.

Key words: rMOMP protein; Rat intraocular hypertension; Retinal optic nerve injury; Immune protection

\section{INTRODUCTION}

The duration of an immunological effect induced by subcutaneous injection with rMOMP protein vaccine is relatively short, but glaucoma and other retinal optic nerve injuries require long-term treatment. Therefore, this method is obviously limited and therapeutically unsatisfactory with a certain toxic effect and the risk of inducing EAE (Dickson and Zurawski, 2010). Therefore, it is imperative to change the route of inoculation and adjuvants to improve the applicability of the vaccine. Conjunctiva-associated lymphoid tissue is mainly located in the folds of lower palpebral conjunctiva. The vessels in the palpebral conjunctiva have intensive distribution and rich blood supply. There are two mesh lymphatic systems in superficial and deep layers, respectively, which are crucial local mucosal-associated lymphoid tissues (Akasaka et al., 2012; McCollum et al., 2012; Saito et al., 2012). Glaucoma and optic nerve injury can lead to progressive death of retinal ganglion cells (RGCs) and loss of optic nerve fibers, which is one of the essential reasons for such diseases inducing blindness (BrignoleBaudouin et al., 2012).

This study aimed to prepare rMOMP protein ophthalmic vaccine in the form of ocustilla. Once being prepared successfully, it has unique advantages: 1) vaccine antigen ingredients can directly stimulate conjunctival mucosal-associated lymphocytes, identify and present the cells and generate immunological effects; 2) vaccine may be absorbed by the cornea to stimulate retinal microglial cells and other antigen-presenting cells, and to generate immunological effects; 3 ) it can generate local immune reaction, reducing the risk of complications induced by systemic immune response; 4) it is painless and non-invasive and is prone to being accepted by patients through the inoculation route of eye drops; 5) it prevents the use of needles and syringes, which can introduce pollution, as well as the transmission of hepatitis B and C and HIV by blood (iatrogenic transmission); 6) its vaccination operation is convenient without the need of healthcare professionals (Filbin, 2008; Abdesselem et al., 2009; Su et al., 2009).

\section{MATERIAL AND METHODS}

\section{Preparation of rMOMP-CS ophthalmic vaccine}

rMOMP protein solution $(2 \mathrm{~mL}, 1 \mathrm{mg} / \mathrm{mL}$ ) was added to $5 \mathrm{~mL}$ double-distilled water to prepare a $0.2-\mathrm{mg} / \mathrm{mL}$ solution, which was sterilized through a $0.22-\mu \mathrm{m}$ sterile Millipore filter repeatedly. Under sterile conditions, $5 \mathrm{mg} / \mathrm{mL}$ chitosan solution and rMOMP protein solution were mixed in equal volumes and stirred sufficiently. The $\mathrm{pH}$ was then adjusted to 7.0 by $0.5 \mathrm{M}$ sodium hydroxide, and the solution was referred to as rMOMP-CS ophthalmic vaccine, which was stored at $4^{\circ} \mathrm{C}$. 


\section{Establishment of animal models}

Sixty normal adult SD rats (30 males and 30 females), aged 9 to 11 weeks old and weighing 200-210 g were selected, and provided by the Experimental Animal Center of the Institute of Field Surgery, Daping Hospital, Third Military Medical University. The 60 rats were randomly divided into two groups to establish the chronic ocular hypertension model and the optic nerve injury model. Chronic ocular hypertension model: 532-diode laser photocoagulation was performed on the trabecular meshwork and superior temporal and infratemporal sclera superficial vein of SD rats. The intraocular pressure of test animals was determined with a TONO-PenXL ophthalmotonometer. Optic nerve injury model: palpebra superior and superior conjunctiva and extraocular muscle (OD) were cut off to expose the optic nerve which was then clamped with a 30-g bulldog clamp for $1 \mathrm{~min}$ at $2 \mathrm{~mm}$ behind the eyeball, the wound was then sutured, the conjunctival sac was coated with chlortetracycline eye ointment and 20,000 U penicillin were inject intramuscularly (Yuen and Wong, 2012).

\section{Inoculation of rMOMP-CS ophthalmic vaccine}

Thirty rats in each group were randomly divided into 2 groups: rMOMP-CS group and $\mathrm{CS}$ group, $\mathrm{N}=15$.

The rats in the rMOMP-CS group were inoculated with rMOMP-CS ophthalmic vaccine by eye drops. The steps were as follows: at the lateral oblique upward side of the right eye, the double eyelids were gently lifted to drop the rMOMP-CS ophthalmic vaccine in the conjunctival sac, and the eyelids were then closed passively. Eye drops were applied once on the 10th and 20th day three times in total to strengthen the immune effect. The rats in the CS group were subjected to the same vaccination strategy. The model rats were sampled on the 20 th day after the final vaccination.

\section{Retrograde tracing of RGCs}

Five experimental rats were randomly selected from each group 5 days before sampling, which were anesthetized by intraperitoneal injection with $1.0 \mathrm{~mL} / \mathrm{kg} 2 \%$ pentobarbital sodium. The rat was fixed on the stereotaxic apparatus and disinfected, the skin was cut off along the parietal midline, and $10 \%$ hydrogen peroxide was smeared at the cranial suture to identify the Bregma point. The stereotaxic apparatus was used to determine the position of the superior colliculus and lateral geniculate body. In the corresponding position, a dental drill was used to open the skull, and the bilateral superior colliculus and lateral geniculate body were slowly injected with $2 \mu \mathrm{L} 2 \%$ FG via the microsyringe, which was completed within 1 $\mathrm{min}$. The osseous reamer was closed with artificial bone meal and the skin was sutured. Penicillin $(20,000 \mathrm{U})$ was injected intramuscularly. Three days later, a catheter was intubated in the ventriculus sinister-aorta, the vessel was ligated and the catheter was fixed. The auricula dextra was the cut off, and normal saline was injected through the conduit to drain the blood from the cor dextrum until transparent normal saline drained. Normal saline was replaced with $100 \mathrm{~mL} \mathrm{2 \%}$ paraformaldehyde for grouting to make the limbs of SD rats straight and stiff. The left and right eyeballs were disconnected. The cornea was cut off along the limbal cornea to remove the crystalline lens and vitreous body which then were put into $2 \%$ paraformaldehyde for overnight fixing. The retina was peeled off carefully to face the vitreous body upward and 
pave it on the glass slide subjected to poly-l-lysine treatment. The slide was then mounted with $70 \%$ glycerol. RGCs were observed with a laser scanning confocal microscope (excitation wavelength: $354 \mathrm{~nm}$ ) and counted.

\section{Detection of retinal GAP43, CD3, BDNF, and GDNF expressions by immunofluorescence}

Five experimental rats were randomly selected from each group, which were anesthetized by intraperitoneal injection with $1.0 \mathrm{~mL} / \mathrm{kg} 2 \%$ pentobarbital sodium. A catheter was intubated in the ventriculus sinister-aorta, the vessel was ligated and the catheter was fixed. The auricula dextra was cut off, and normal saline was injected through the conduit to drain the blood from the cor dextrum until transparent normal saline drained. Normal saline was replaced with $100 \mathrm{~mL} \%$ paraformaldehyde for grouting to make the limbs of SD rat straight and stiff. The left and right eyeballs were taken out, embedded in OCT gel and quickly frozen for frozen sections with a thickness of $5 \mu \mathrm{m}$. The sections were mounted on glass slides subjected to poly-l-lysine treatment, and stationary liquid was then added dropwise. The slides were allowed to stand at room temperature for $30 \mathrm{~min}$, and stored at $4^{\circ} \mathrm{C}$ after the stationary liquid was poured off. The slides were washed with 0.2 M PBS, pH 6.9-7.1, for 3 min, 3 times, made transparent with $0.3 \%$ Triton X-100 for 15 min, washed with $0.2 \mathrm{M}$ PBS, pH 6.9-7.1, 3 times, 3 min each time, or with $0.02 \mathrm{M}$ citrate, $\mathrm{pH} 6.5$, at $92^{\circ} \mathrm{C}$ for $15 \mathrm{~min}$, cooled naturally, then washed with $0.2 \mathrm{M}$ PBS, pH 6.9-7.1, 3 times, 3 min each time, and blocked with $10 \%$ BSA for $30 \mathrm{~min}$, which was then poured out. The primary antibody at 1:100 dilution was added dropwise, and the slides placed at $4^{\circ} \mathrm{C}$ overnight and washed with $0.2 \mathrm{M}$ PBS, pH 6.9-7.1, for 3 min, 3 times. The secondary antibody diluted 1:100 was added dropwise, and the slides were incubated in a $37^{\circ} \mathrm{C}$ incubator for $3 \mathrm{~h}$ and washed with $0.2 \mathrm{M} \mathrm{PBS}, \mathrm{pH}$ 6.9-7.1, 3 times, 3 min each time. DAPI chromogenic agent working solution was then added dropwise and the slides placed at room temperature for $10 \mathrm{~min}$ and washed with $0.2 \mathrm{M}$ PBS, $\mathrm{pH}$ 6.9-7.1, three times, 3 min each time. The anti-fluorescent quenching mounting medium was used to mount slides which were then observed with a laser scanning confocal microscope.

\section{RESULTS}

\section{Characters}

The vaccine was a light yellow, slightly viscous clear liquid.

\section{pH, osmotic pressure and viscosity}

The $\mathrm{pH}$, osmotic pressure and viscosity of the vaccine are shown in Table 1.

Table 1. $\mathrm{pH}$, osmotic pressure and viscosity of the vaccine.
\begin{tabular}{lcccc}
\hline Sample No. & $\mathrm{pH}$ & Osmotic pressure (MOsm) & Viscosity (mPa·s) \\
\cline { 2 - 5 } & & rMOMP-CS & $0.9 \% \mathrm{NaCl}$ & 301 \\
\hline 1 & 7.1 & 285 & 298 & 4.43 \\
2 & 7.2 & 286 & 300 & 4.28 \\
3 & 7.1 & 288 & 299.67 & 4.36 \\
Mean & 7.13 & 286.33 & & \\
\hline
\end{tabular}




\section{Retrograde tracing of RGCs by fluorogold}

The results showed that the number of RGCs of the rMOMP-CS group in the chronic ocular hypertension model was significantly higher than that of the CS group $(\mathrm{P}<0.05)$. The RGC number of the rMOMP-CS group in the optic nerve clamping injury model was higher than that of the CS group with the difference statistically significant $(\mathrm{P}<0.01)$. The results show that after the rats in the two retinal optic nerve injury models were inoculated with rMOMP-CS vaccine. RGCs were increased, indicating that rMOMP-CS vaccine reduced the death of RGCs after retinal optic nerve injury and increased the survival rate of RGCs, thus protecting the retinal optic nerve (Figure 1, Table 2).

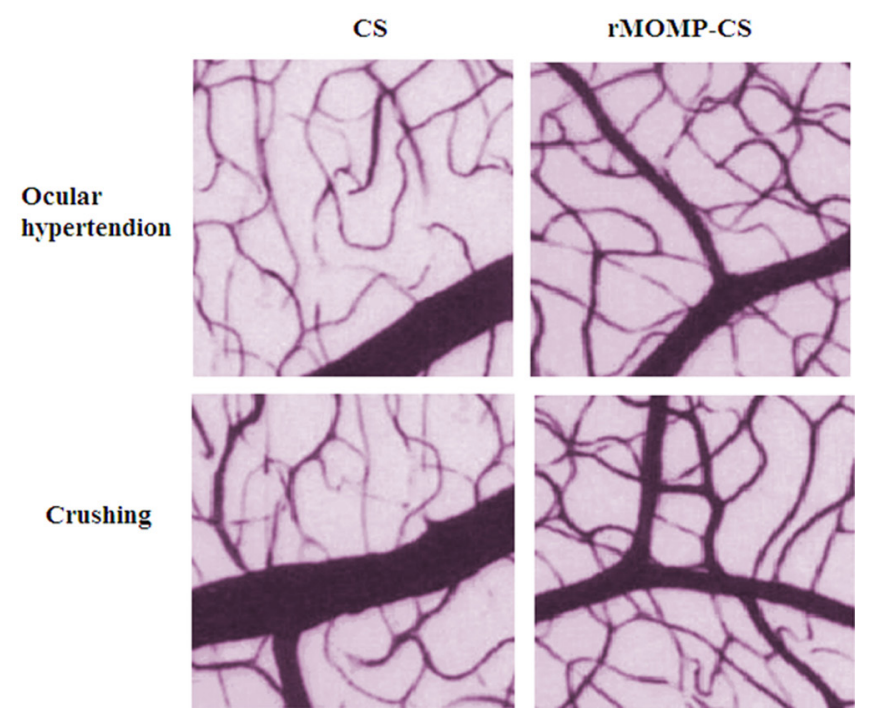

Figure 1. RGCs retrograde FG staining after the inoculation of rMOMP-CS vaccine (400X).

Table 2. RGCs number after the inoculation of rMOMP-CS vaccine (400X).

\begin{tabular}{lcrr}
\hline & Normal & rMOMP-CS & CS \\
\hline Chronic ocular hypertension & $2237.41 \pm 47.52^{*}$ & $2014.07 \pm 51.36$ & $1820.00 \pm 51.31^{*}$ \\
Optic nerve clamping injury & $2198.53 \pm 37.14^{*}$ & $1814.12 \pm 54.00$ & $1613.24 \pm 51.67^{*}$ \\
\hline
\end{tabular}

Compared with the rMOMP-CS group of the same model rats, $* \mathrm{P}<0.05$.

\section{Retinal GAP43 expression}

A great increase in GAP43 expression was found in the retinal layers of the rMOMPCS group in the chronic ocular hypertension model. No expression was found in the CS group (Figure 2).

A great increase in GAP43 expression was found in the retinal layers of the rMOMPCS group in the optic nerve clamping injury model; no expression was found in the CS group (Figure 3). 


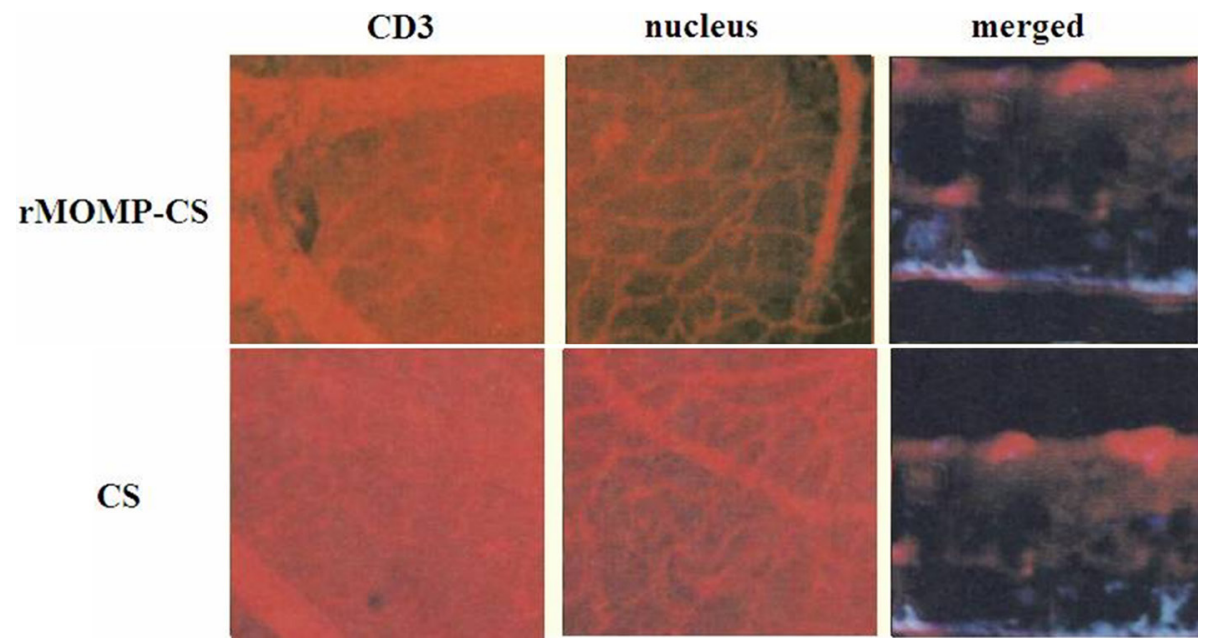

Figure 2. Retinal GAP43 expression in the chronic ocular hypertension model rats after inoculation of rMOMP-CS vaccine.

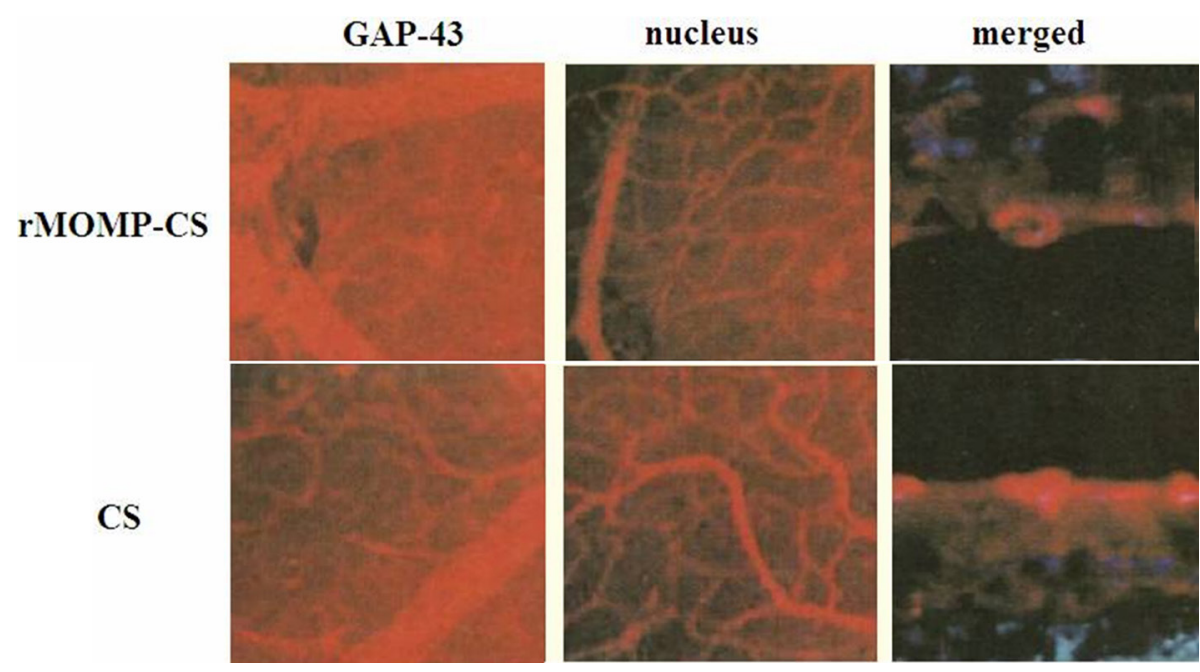

Figure 3. Retinal GAP43 expression in the optic nerve clamping injury model rats after inoculation of rMOMP-CS vaccine.

\section{Retinal CD3 expression}

CD3 expression was evident in the ganglion cell layer, inner plexiform layer and internal and external nuclear layers of the retina of the rMOMP-CS group in the chronic ocular hypertension model; no CD3 expression was found in the retina of the CS group (Figure 4).

CD3 expression was evident in the ganglion cell layer, inner plexiform layer and internal and external nuclear layers of the retina of the rMOMP-CS group in the optic nerve clamping injury model; no CD3 expression was found in the retina of the CS group (Figure 5). 


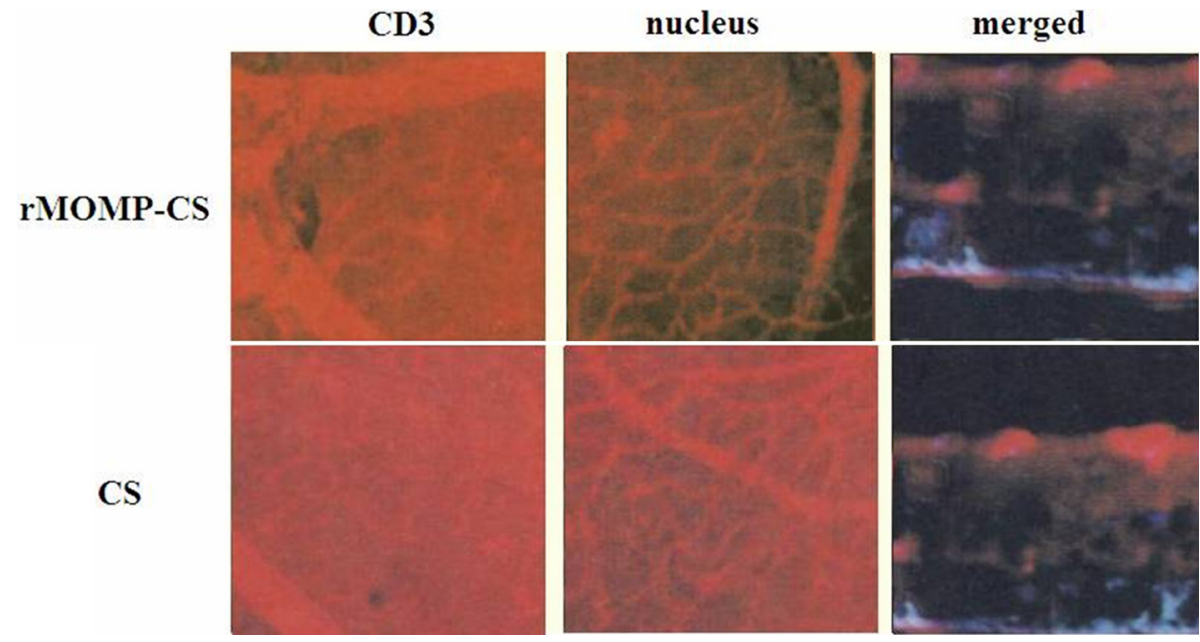

Figure 4. Retinal CD3 expression in the chronic ocular hypertension model rats after inoculation of rMOMP-CS vaccine.

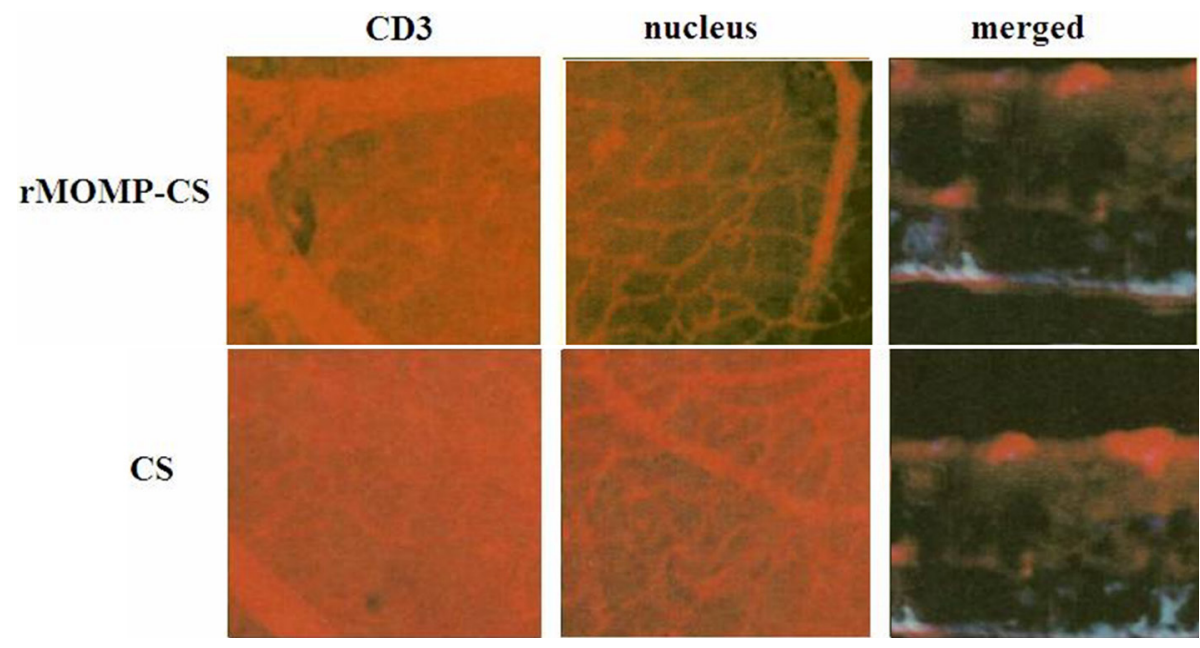

Figure 5. Retinal CD3 expression in the optic nerve clamping injury model rats after inoculation of rMOMP-CS vaccine.

\section{Retinal GDNF and BDNF expressions}

GDNF and BNDF were abundantly expressed in retinal layers in the rMOMP-CS group in the chronic ocular hypertension model. A small amount of GDNF expression could be found in the retina of the CS group, which was mainly distributed in the inner plexiform layer. Immunoblotting results showed that the expression of BDNF and GDNF in the rMOMP$\mathrm{CS}$ group was significantly higher than that of the CS group $(\mathrm{P}<0.05)$ (Figure 6). 

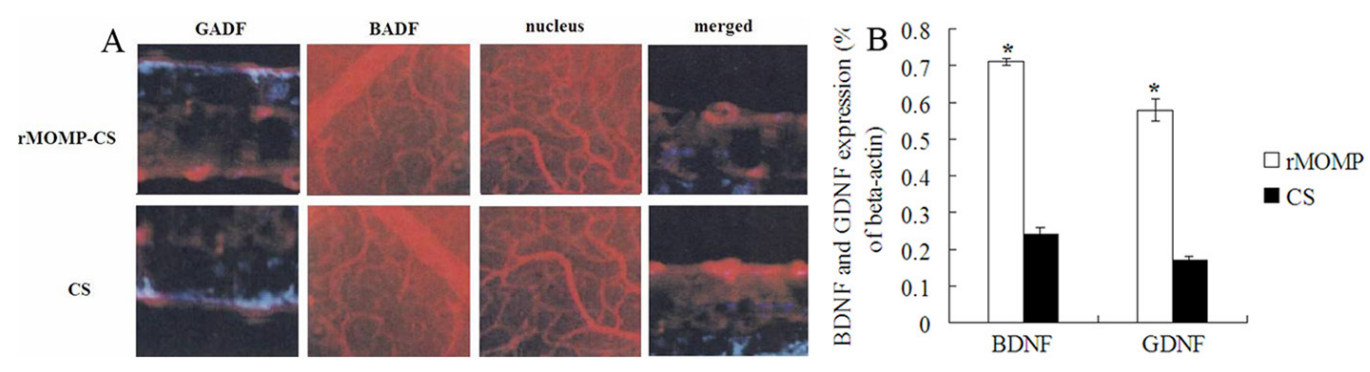

Figure 6. Retinal BADF and GADF expressions in the chronic ocular hypertension model rats after inoculation of rMOMP-CS vaccine. A. Immunofluorescence assay; B. histogram analysis (compared with the CS group, ${ }^{*} \mathrm{P}<0.05$ ).

GDNF and BNDF were abundantly expressed in retinal layers in the rMOMP-CS group in the optic nerve clamping injury model, which were mainly distributed in the ganglion cell layer, inner plexiform layer and inner nuclear layer; a small amount of GDNF and BNDF expression was found in the retina of the CS group. Immunoblotting results showed that the expression of BDNF and GDNF in the rMOMP-CS group was significantly higher than that of the CS group $(\mathrm{P}<0.05)$ (Figure 7).

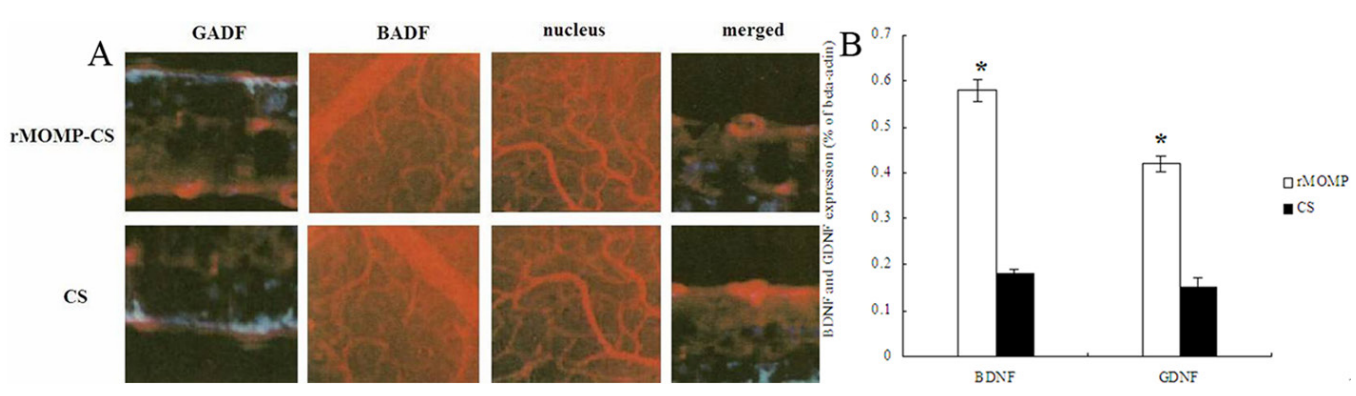

Figure 7. Retinal BADF and GADF expressions in the optic nerve clamping injury model rats after inoculation of rMOMP-CS vaccine. A. Immunofluorescence assay; B. histogram analysis (compared with the CS group, $* \mathrm{P}<0.05$ ).

\section{DISCUSSION}

rMOMP, an important molecule isolated from myelin in the central nervous system, exhibits an inhibitory role in nerve regeneration. rMOMP is a structural domain with neural inhibitory activity, which can induce the collapse of the growth cone of damaged axons (Sun et al., 2012). It has been confirmed that active immunization with myelin protein antigen vaccine or passive transfer of T cells and specific antibodies stimulated by this antigen can promote the regeneration of damaged nerves. Active immunization of myelin-derived peptides or passive transfer of anti-myelin T cells in rats can induce a neuroprotective response in the optic nerve clamping injury model. Smith et al. (2012) injected with spinal cord homogenate vaccine three times in the optic nerve tiny crush injury model of SD rats and found that axons could pass through the site of injury and be regenerated, and compared with the liver vaccine control 
group, this method could promote axonal regeneration by $250 \mu \mathrm{m}$ on average (Funderud et al., 2012; Karlsson et al., 2012). Smith et al. (2012) vaccinated animals with Cop-1 peptide and found that Cop-1 peptide could activate T lymphocyte-mediated neuroprotective effects in the early stage; through the observation of RGCs 3 weeks after intraocular hypertension, they found that Cop-1 peptide could effectively reduce the loss of RGCs caused by intraocular hypertension (Donegan and Lieberman, 2012; Smith et al., 2012). In vivo and in vitro tests also confirmed that hybridoma cells were implanted with anti-rMOMP monoclonal antibody IN-1 or IN-1Fab fragment in impaired site shortly after spinal cord injury, damaged corticospinal tract appeared signs of regeneration, and regenerating fibers in the distal impaired site formed dense branches to re-establish connection with partial spinal neuronal loops (Pernet and Schwab, 2012; Wang and Xiong, 2012). IN-1 cannot only promote the regeneration of injured nerve fibers but can also facilitate the sprouting and plasticity growth of undamaged plane motor nerve fiber.

Physiological enhancement of $\mathrm{T}$ lymphocytes after the injury of the optic nerve and other central nervous systems may mediate protective autoimmunity, which may be related to the activation of $\mathrm{T}$ lymphocytes to produce nerve growth factors, brain-derived neurotrophic factors, neurotrophin-3, neurotrophin- $4 / 5$ and other nutritional factors and the enhancement of scavenging effect of macrophages to remove harmful substances. Blocking inhibitory molecules generated by autoimmune antibodies produced or other pathways that have not yet been elucidated can also lead to a neuroprotective effect (Brambilla et al., 2012; Das et al., 2012; Shaw and Kelley 2012). Therefore, this study screened the best concentration of rMOMP protein vaccine to activate the immune system of rats to the greatest degree, so that the proliferation of $\mathrm{T}$ lymphocytes was significantly enhanced and that the specific IgG antibody titers were significantly increased. It is possible to achieve protection for the injured nerve through above approaches and promote nerve regeneration.

The common pathological basis of glaucoma and optic nerve injury is the irreversible death and difficult regeneration of RGCs, further leading to the atrophy of the optic nerve and loss of function (Tanito et al., 2012; Wang et al., 2012). Therefore, the survival rate of RGCs was taken as the main indicator in this study. After rMOMP protein ophthalmic vaccine was injected into the rats of the chronic ocular hypertension model and optic nerve clamping injury model, it was observed that although the number of retinal RGCs, was not as much as that of uninjured rats, it was significantly increased compared with the control group of CS vaccination model, indicating that rMOMP protein ophthalmic vaccine can protect RGCs, reducing their death and increasing their survival.

The experimental results showed that rMOMP protein ophthalmic vaccine can promote the infiltration of retinal reactive $\mathrm{T}$ cells in rats with retinal optic nerve injury and induce retinal microglia activation. Studies have confirmed that autoimmune T cells aggregated in CNS damaged parts can generate a variety of neurotrophic factors, and that microglial cells also release a small amount of neuroprotective factors in the process of activation (Howell et al., 2012). Therefore, we speculate that mainly GDNF, BDNF and other neurotrophic factors that are secreted by the active $\mathrm{T}$ cells infiltrated into the retina and activated retinal microglial cells to enhance the above initiative neuron survival process, improve the micro environment of RGCs at the site of retinal optic nerve injury, reduce the secondary damage of RGCs, and promote the regeneration of RGCs, increasing the survival rate of RGCs. 


\section{REFERENCES}

Abdesselem H, Shypitsyna A, Solis GP, Bodrikov V, et al. (2009). No rMOMP- and NgR-mediated inhibition of regenerating axons in the zebrafish optic nerve. J. Neurosci. 29: 15489-98.

Akasaka R, Chiba T, Dutta AK, Toya Y, et al. (2012). Colonic mucosa-associated lymphoid tissue lymphoma. Case Rep. Gastroenterol. 6: 569-575.

Brambilla R, Dvoriantchikova G, Barakat D, Ivanov D, et al. (2012). Transgenic inhibition of astroglial NF-kappaB protects from optic nerve damage and retinal ganglion cell loss in experimental optic neuritis. J. Neuroinflammation 9: 213-219.

Brignole-Baudouin F, Desbenoit N, Hamm G, Liang H, et al. (2012). A new safety concern for glaucoma treatment demonstrated by mass spectrometry imaging of benzalkonium chloride distribution in the eye, an experimental study in rabbits. PLoS One 7: 180-188.

Das A, Guyton MK, Smith A, Wallace G IV, et al. (2012). Calpain inhibitor attenuated optic nerve damage in acute optic neuritis in rats. $J$. Neurochem. 18: 585-560.

Dickson HM and Zurawski J (2010). POSH is an intracellular signal transducer for the axon outgrowth inhibitor rMOMP. J. Neurosci. 30: 13319-13325.

Donegan RK and Lieberman RK (2012). New direction for glaucoma therapeutics: focus on the olfactomedin domain of myocilin. Future Med. Chem. 4: 2131-2134.

Filbin MT (2008). PirB, a second receptor for the myelin inhibitors of axonal regeneration rMOMP, MAG, and OMgp: implications for regeneration in vivo. Neuron 60: 740-742.

Funderud I, Lindgren M, Løvstad M, Endestad T, et al. (2012). Differential Go/rMOMP Activity in Both Contingent Negative Variation and Spectral Power. PLoS One 7: 504-510.

Howell GR, Soto I, Zhu X, Ryan M, et al. (2012). Radiation treatment inhibits monocyte entry into the optic nerve head and prevents neuronal damage in a mouse model of glaucoma. J. Clin. Invest. 122: 1246-1261.

Karlsson TE, Karlén A, Olson L and Josephson A (2012). Neuronal overexpression of rMOMP receptor 1 in APPswe/ PSEN1(DeltaE9) mice impairs spatial cognition tasks without influencing plaque formation. J. Alzheimers Dis. 33: 145-155.

McCollum CR, VanAsselberg CB, Cook-Glen CL, Bhagat R, et al. (2012). Endobronchial recurrence of gastric mucosaassociated lymphoid tissue lymphoma. J. Bronchol. Interv. Pulmonol. 19: 338-339.

Pernet V and Schwab ME (2012). The role of rMOMP-A in axonal plasticity, regrowth and repair. Cell Tissue Res. 349: 97-104.

Saito Y, Suzuki H, Tsugawa H, Imaeda H, et al. (2012). Overexpression of miR-142-5p and miR-155 in Gastric MucosaAssociated Lymphoid Tissue (MALT) Lymphoma Resistant to Helicobacter pylori Eradication. PLoS One 7: 396.

Su Y, Wang F, Teng Y, Zhao SG, et al. (2009). Axonal regeneration of optic nerve after crush in rMOMP receptor knockout mice. Neurosci. Lett. 460: 223-226.

Sun HH, Gao F, Liu B, Yu HT, et al. (2012). Inhibition of rMOMP expression to promote repair after spinal cord injury. Chin. Med. J. 125: 4044-4048.

Shaw ML and Kelley B (2012). Collateral damage: heat transfer as a possible mechanism of optic nerve injury during neurosurgical intervention. Ophthal. Plast. Reconstr. Surg. 28: 328-330.

Smith ND, Crabb DP, Glen FC, Burton R, et al. (2012). Eye movements in patients with glaucoma when viewing images of everyday scenes. Seeing Perceiving 25: 471-492.

Tanito M, Kaidzu S, Takai Y and Ohira A (2012). Status of systemic oxidative stresses in patients with primary open-angle glaucoma and pseudoexfoliation syndrome. PLoS One 7: 680-687.

Wang D, Huang Y, Huang C, Wu P, et al. (2012). Association analysis of cigarette smoking with onset of primary openangle glaucoma and glaucoma-related biometric parameters. BMC Ophthalmol. 12: 59-64.

Wang T and Xiong JQ (2012). The role of rMOMP-A in neuroregeneration: a review. Brain Res. Bull. 87: 499-503.

Yuen NS and Wong IY (2012). Congenital glaucoma from Sturge-Weber syndrome: a modified surgical approach. Korean J. Ophthalmol. 26: 481-484. 